TRANSACTIONS OF THE

AMERICAN MATHEMATICAL SOCIETY

Volume 357, Number 2, Pages 735-756

S 0002-9947(04)03562-7

Article electronically published on April 27, 2004

\title{
ON THE COHEN-MACAULAY MODULES OF GRADED SUBRINGS
}

\author{
DOUGLAS HANES
}

\begin{abstract}
We give several characterizations for the linearity property for a maximal Cohen-Macaulay module over a local or graded ring, as well as proofs of existence in some new cases. In particular, we prove that the existence of such modules is preserved when taking Segre products, as well as when passing to Veronese subrings in low dimensions. The former result even yields new results on the existence of finitely generated maximal Cohen-Macaulay modules over non-Cohen-Macaulay rings.
\end{abstract}

\section{INTRODUCTION AND DEFINITIONS}

The notion of a linear maximal Cohen-Macaulay module over a local ring $(R, m)$ was introduced by Ulrich [17], who gave a simple characterization of the Gorenstein property for a Cohen-Macaulay local ring possessing a maximal Cohen-Macaulay module which is sufficiently close to being linear, as defined below. A maximal Cohen-Macaulay module (abbreviated MCM module) over $R$ is one whose depth is equal to the Krull dimension of the $\operatorname{ring} R$. All modules to be considered in this paper are assumed to be finitely generated. The minimal number of generators of an $R$-module $M$, which is equal to the $(R / m)$-vector space dimension of $M / m M$, will be denoted throughout by $\nu(M)$. In general, the length of a finitely generated Artinian module $M$ is denoted by $\ell(M)$ (or by $\ell_{R}(M)$, if it is necessary to specify the ring acting on $M$ ). If the dimension of the module $M$ is $d$, then the multiplicity of $M$ with respect to an $m$-primary ideal $I$ may be defined as

$$
e(I ; M)=(d !) \cdot \lim _{t \rightarrow \infty} \frac{\ell\left(M / I^{t} M\right)}{t^{d}} .
$$

Alternatively, $e(I ; M) / d$ ! is the leading coefficient of an appropriate Hilbert polynomial of $M$. We usually just write $e(M)$ for the multiplicity of $M$ with respect to the maximal ideal $m$, and refer to $e(M)$ as the multiplicity of the module $M$.

Definition 1.1. Let $(R, m)$ be a Noetherian local ring with a finitely generated maximal Cohen-Macaulay module $M$. Then $M$ is said to be a linear maximal Cohen-Macaulay module (or a maximally generated maximal Cohen-Macaulay module) if $e(M)=\nu(M)$.

It is easy to see that the condition for linearity is a maximality condition, in the sense that, for any MCM $R$-module $M$, one has $\nu(M) \leq e(M)$.

Received by the editors November 11, 2001 and, in revised form, August 14, 2003.

2000 Mathematics Subject Classification. Primary 13C14.

(C)2004 American Mathematical Society 
The first existence results for linear MCMs have been recorded by Brennan, Herzog, and Ulrich 2, who prove the existence of linear maximal Cohen-Macaulay modules for one-dimensional rings, two-dimensional graded Cohen-Macaulay domains, rings of minimal multiplicity, and certain rings of determinantal varieties. The most significant existence result to appear subsequently is that of Backelin, Herzog, and Ulrich 1], who prove the existence of linear maximal Cohen-Macaulay modules over local rings that are strict complete intersections (i.e., both the ring and its associated graded ring are complete intersections). The question of whether general complete intersection rings possess linear MCM modules remains open.

Ulrich has even posed the question of whether every local Cohen-Macaulay ring posesses a linear MCM module [17. Although there is no known counterexample, the condition is so strong as to make a positive answer seem unlikely in general. However, the existence of linear maximal Cohen-Macaulay modules in any particular case has very strong consequences, as will be seen below.

First, we give Ulrich's characterization of Gorenstein rings. Theorem 1.2 implies that, in the presence of a linear MCM $M$, the Gorenstein property is equivalent to the vanishing of the modules $\operatorname{Ext}_{R}^{i}(M, R)$ for $0<i \leq \operatorname{dim}(R)$. Thus the existence of a linear MCM module may greatly simplify the determination of the Gorenstein property.

Theorem 1.2 (Ulrich). Let $(R, m)$ be a Cohen-Macaulay local ring, and suppose that $R$ possesses a finitely generated module $M$ with the property that $2 \nu(M)>$ $e(M)$. Then $R$ is Gorenstein if $\operatorname{Ext}_{R}^{i}(M, R)=0$ for $0<i \leq \operatorname{dim}(R)$.

In addition to this result, it is known that the existence of linear MCM modules over any class of Noetherian local rings implies the existence of finitely generated MCM modules over their associated graded rings. In fact, it is shown in 2] that if $(R, m)$ is a homomorphic image of a regular local ring $(S, n)$, then an $R$-module $M$ is a linear MCM module if and only if $M$ has linear resolution of length $\operatorname{dim}(S)-\operatorname{dim}(R)$ over $S$ (hence the name). (A finite resolution is called linear if an appropriate associated graded resolution, defined by matrices of linear forms, is exact.) It follows that the associated graded module is resolved by the associated graded resolution, and is therefore a maximal Cohen-Macaulay module (and in fact linear). This is of great interest, since the associated graded ring of even a Cohen-Macaulay ring need not be Cohen-Macaulay (it can even have depth zero), and since the existence of finitely generated MCM modules remains a major open question in commutative algebra, even for finitely generated graded algebras over a field (cf. 11]).

Finally, the existence of a linear MCM module over the base ring $R$ implies the following longstanding conjecture of Lech 13 on the multiplicities of a faithfully flat extension of local rings:

Conjecture 1.3 (Lech). Let $(R, m) \subseteq(S, n)$ be a faithfully flat extension of Noetherian local rings. Then $e(R) \leq e(S)$.

A discussion of this implication, as well as some new results on the conjecture, can be found in [8, 9].

In this paper we will prove that the existence of a linear MCM module can be preserved when taking the Segre product of algebras over a field (section 2) or, in low dimensions, when passing to a Veronese subring (section 3). Our results on Segre products imply the existence of finitely generated MCM modules over 
many non-Cohen-Macaulay graded algebras (see Corollary 2.7). The results on Veronese subrings, while more limited in themselves, have significant generalizations to standard graded algebras of prime characteristic (section 4).

Many of the results of this article have appeared in preliminary form in the author's Ph.D. thesis [8]. Recently, Eisenbud and Schreyer [6] have generalized several results of this article, especially those regarding Veronese subrings. While Eisenbud and Schreyer were aware of our earlier work, their approach is different and in some ways far more general.

1.1. Characterizations. We may characterize the linearity property in several different ways. For example, if we assume that the residue field of the $d$-dimensional local ring $(R, m)$ is infinite, then we may choose a minimal reduction $I=\left(x_{1}, \ldots, x_{d}\right)$ of $m$ [15], generated by a system of parameters. We then know that for any MCM module $M, e(M)=\ell(M / I M)$ and $\nu(M)=\ell(M / m M)$. This makes it clear that $e(M) \geq \nu(M)$ (the general case of this inequality can be deduced by extending the residue field) and that $M$ is linear if and only if $m M=I M$. Moreover, we see that the Hilbert function of a linear MCM module $M$ must be given by

$$
H_{M}(t)=\operatorname{dim}_{R / m}\left(m^{t} M / m^{t+1} M\right)=\left(\begin{array}{c}
t+d-1 \\
d-1
\end{array}\right) \cdot \nu(M),
$$

the Hilbert function of a free module over a regular ring of dimension $d$. This characterization of linearity will be stressed in much of the work to be presented here, and lends itself to the following natural generalization:

Definition 1.4. The reduction degree of a finitely generated MCM module $M$ over a local ring $(R, m)$ is the least integer $t$ such that $m^{t} M \subseteq I M$ for some ideal $I$ generated by a system of parameters of $R$.

Remark 1.5. Of course, we are generally thinking of the parameter ideal $I$ as being a minimal reduction. When $R$ does not necessarily possess minimal reductions, it will usually be desirable to pass to a flat extension with infinite residue field before applying the definition.

A second formulation of the existence of linear MCM modules is as follows: assume that $R$ is either a complete local ring with infinite residue field $K$, or else is a standard graded algebra over a field $K$. Then we may choose a minimal reduction $\left(x_{1}, \ldots, x_{d}\right)$ of the maximal ideal $m$ and express $R$ as a module-finite extension of either the power series ring $A=K\left[\left[x_{1}, \ldots, x_{d}\right]\right]$ (in the complete case) or of the polynomial ring $A=K\left[x_{1}, \ldots, x_{d}\right]$ (in the graded case). A MCM module $M$ over $R$ must also be MCM, hence free, over the regular ring $A$. Thus, a MCM module over $R$ is simply a free $A$-module with the added structure of an $R$-module, and the existence of a MCM $R$-module is equivalent to the existence of an $A$-algebra homomorphism $R \rightarrow \mathcal{M}_{n}(A)$, the ring of $n \times n$ matrices over $A$, for some positive integer $n$ [10. We should note that, if the module $M$ is to have positive rank over $R$, then

$$
n=\operatorname{rank}_{A}(M)=\operatorname{rank}_{A}(R) \cdot \operatorname{rank}_{R}(M)=e(R) \cdot \operatorname{rank}_{R}(M) .
$$

Thus the size $n$ of the matrices must be divisible by the multiplicity $e(R)$.

The existence of a linear MCM module is similarly characterized by the following lemma. Throughout the paper, we shall refer to an affine $\mathbb{N}$-graded algebra over a field $K$, generated by forms of degree one, as a standard graded $k$-algebra. One 
should recall that, if $(R, m)$ is any local ring, then the associated graded $\operatorname{ring} \operatorname{gr}_{m}(R)$ is a standard graded algebra over the residue field $R / m$.

Lemma 1.6. Let $(R, m)$ be a complete local ring of equal characteristic, with coefficient field $K$, or else a standard graded algebra over $K$, and let $I=\left(x_{1}, \ldots, x_{d}\right)$ be a minimal reduction of the maximal ideal $m$. Set $A=K\left[\left[x_{1}, \ldots, x_{d}\right]\right] \subseteq R$ (or $A=K\left[x_{1}, \ldots, x_{d}\right]$ in the graded case). Then $R$ has a linear MCM module if and only if there exists, for some $n>0$, an A-algebra homomorphism $R \rightarrow \mathcal{M}_{n}(A)$ such that all elements of $m$ map to matrices with all entries in the maximal ideal $\left(x_{1}, \ldots, x_{d}\right)$ of $A$. In the graded case, one may further assume that the generators of the homogeneous maximal ideal $m$ map to matrices whose entries are linear forms in $x_{1}, \ldots, x_{d}$.

Proof. The existence of the map $R \rightarrow \mathcal{M}_{n}(A)$ corresponds to $A^{n}$ having the structure of a MCM $R$-module, as seen above. This module $M$ is linear if and only if $m M=I M$, which means that elements of $m$ act on $A^{n}$ by matrices with entries in $I$.

In the graded case, we extend the system of parameters $x_{1}, \ldots, x_{d}$ to a minimal generating set $x_{1}, \ldots, x_{r}$ of $m$. Assume that for each $d+1 \leq i \leq r$, the generator $x_{i}$ maps to

$$
B_{i}=B_{i 1}+B_{i 2}+B_{i 3}+\ldots+B_{i t_{i}}
$$

where $B_{i j}$ is an $n \times n$ matrix whose entries are forms of degree $j$ in $A$. That this gives an $A$-algebra homomorphism is equivalent to the matrices $B_{i}$ satisfying certain polynomial equations over $A$; the same as those satisfied by the generators of $m$, plus degree two homogeneous equations ensuring commutivity. All of these polynomials are of the form

$$
G_{j}\left(x_{1}, \ldots, x_{d}, Z_{d+1}, \ldots, Z_{r}\right) \in A\left[Z_{d+1}, \ldots, Z_{r}\right],
$$

where $G_{j}$ is homogeneous with respect to the maximal ideal

$$
\left(x_{1}, \ldots, x_{d}, Z_{d+1}, \ldots, Z_{r}\right) \text {. }
$$

Now just considering the lowest degree component of the matrix equations

$$
G_{j}\left(x_{1}, \ldots, x_{d}, B_{d+1}, \ldots, B_{r}\right)=0
$$

shows that the degree one components $B_{11}, B_{21}, \ldots, B_{r 1}$ must satisfy the same equations. Thus the generators $u_{i}$ may just as well be mapped to the matrices $B_{i 1}$ of linear forms.

In any case, giving an $A$-algebra homomorphism $R \rightarrow \mathcal{M}_{n}(A)$ corresponds to solving polynomial equations with coefficients in $A$ in some matrix ring $\mathcal{M}_{n}(A)$; namely, the same equations satisfied by the generators of $R$ as an $A$-algebra, plus homogeneous equations of degree two which force the matrices to commute. Each such matrix equation may be rewritten as a set of $n^{2}$ polynomial equations over $A$. Especially in the graded case, this yields a nice characterization of the existence of a linear MCM module:

Proposition 1.7. Let $R$ be a standard graded algebra over a field $K$, let $I=$ $\left(x_{1}, \ldots, x_{d}\right)$ be a minimal reduction of the homogeneous maximal ideal, and let $A=K\left[x_{1}, \ldots, x_{d}\right] \subseteq R$. Then for any $n>0$, there exists a finite system of polynomial equations in finitely many variables over $K$ which admit a solution in $K$ if and only if there exists a linear $M C M R$-module $M$ with $\nu(M)=e(M)=n$. 
Proof. As noted above, the existence of an appropriate module is equivalent to the existence of a solution $\left\{Z_{i}=B_{i} \in \mathcal{M}_{n}\left(A_{1}\right)\right\}$ to finitely many polynomial equations

$$
G_{j}\left(x_{1}, \ldots, x_{d}, Z_{1}, \ldots, Z_{r}\right) \in A\left[Z_{1}, \ldots, Z_{r}\right],
$$

where the $G_{j}$ are homogeneous elements of the ideal $\left(x_{1}, \ldots, x_{d}, Z_{1}, \ldots, Z_{r}\right)$.

Now, if each variable $Z_{i}$ is replaced by an $n \times n$ matrix with indeterminate entries $Z_{i k l}$, each of the equations $G_{j}$ may be replaced by $n^{2}$ homogeneous polynomial equations $G_{j k l}$ in the variables $x_{i}$ and $Z_{i k l}$. The existence of a linear MCM module is now equivalent to a solution of this equation given by setting each of the $r n^{2}$ variables $Z_{i k l}$ equal to a linear form in $A$.

Moreover, if we replace each variable $Z_{i k l}$ by a linear combination

$$
a_{i k l 1} x_{1}+\ldots+a_{i k l d} x_{d},
$$

where the $a_{i k l m}$ are indeterminates over $K$, then comparing coefficients of monomials in $A$ will allow us to replace each equation $G_{j k l}$ by finitely many homogeneous equations on the coordinates $a_{i k l m}$. All of these polynomial equations define a closed subspace of $\mathbb{A}_{K}^{d r n^{2}}$ which is nonempty if and only if there exists a linear MCM module over $R$ with multiplicity $n$.

Thus, the existence of a linear MCM module $M$ with multiplicity $n$ is equivalent to the statement that a certain affine variety contained in $\mathbb{A}_{K}^{d r n^{2}}$ is nonempty. Note that it is not at all clear that one can give a similar characterization of the existence of a (not necessarily linear) MCM module, since there is no way of bounding the degrees of the polynomials which occur as entries in a potential matrix solution. This leads us to believe that the question of which rings possess linear MCM modules may be fundamentally easier to decide than the question of which rings possess finitely generated MCM modules.

All of this is quite closely connected to the following result on linear MCM modules of Brennan, Herzog, and Ulrich [2], which we have actually reproved in the case that $R$ is a standard graded ring with homogeneous maximal ideal $m$ :

Proposition 1.8 (Brennan, Herzog, and Ulrich). If $M$ is a linear MCM module over a local ring $(R, m)$, then the the associated graded module $g r_{m}(M)$ is a finitely generated MCM module over $\operatorname{gr}_{m}(R)$.

This result is interesting in light of the fact that no such statement can be made with regard to a general MCM module over $R$. As mentioned above, it implies the existence of MCM modules over the associated graded rings of any class of local rings for which linear MCM modules can be shown to exist.

\section{Linear MCM modules over Segre Product Rings}

In this section and the next, we will consider two sorts of operations on graded rings under which the existence of linear MCM modules can be shown to persist. Since so little is as yet known with regard to existence, it is natural to consider classes of monomial or determinantal rings, where combinatorial arguments simplify the computation of such numerical invariants as the multiplicity. At least one result in this direction has already been attained [2]:

Proposition 2.1 (Brennan, Herzog, Ulrich). Let $s \geq r \geq 0$ be positive integers, $A=K\left[x_{1}, \ldots, x_{n}\right]$ a polynomial ring over the field $K$, and $C$ an $r \times s m a-$ trix whose entries are linear forms in $A$. Assume, moreover, that grade $\left(I_{r}(C)\right)=$ 
height $\left(I_{r}(C)\right)$, where $I_{r}(C)$ is the ideal of $A$ generated by the $r \times r$ minors of $C$. Then $R=A / I_{r}(C)$ admits a linear MCM module $M$.

We will not reproduce the proof, except to say that the result stated here may be deduced from the case of the ring of generic $r \times r$ minors, where $n=r s$ and $C$ is the matrix which has the variables as its entries. The generic ring is known to be Cohen-Macaulay by a result of Eagon and Northcott [5. In the statement above, the condition on the grade of $I_{r}(C)$ is necessary in order to ensure that $R$ is Cohen-Macaulay.

This result invites the following more general inquiry:

Question 2.2. Let $A=K\left[x_{i j}: 1 \leq i \leq m, 1 \leq j \leq n\right]$ be a polynomial ring over the field $K$, where we assume that $m \leq n$. Let $X$ be the $m \times n$ matrix whose $i j$ entry is $x_{i j}$, and set $R_{r}(X)=A / I_{r}(X)$ for $1 \leq r \leq m$. For which choices of $m, n$, and $r$ does $R_{r}(X)$ admit a linear MCM module?

The results of Eagon and Hochster [4] show that all of the rings $R_{r}(X)$ are CohenMacaulay. The proposition stated above gives a positive answer to the question in the case that $r$ is maximal; namely $r=m$. It will follow from work in this chapter that $R_{2}(X)$ always possesses a linear MCM module (Corollary 2.8). However, we shall not directly concern ourselves with determinantal rings, but instead with Segre product rings. The Segre product of two graded algebras over a field $K$ is defined as follows:

Definition 2.3. Let $R$ and $S$ be positively graded algebras over a field $K$, with $R_{0}=S_{0}=K$. Then the Segre product ring $R \otimes_{\text {seg }} S$ is the positively graded $K$-subalgebra of $R \otimes_{K} S$ with graded pieces $\left(R \otimes_{\text {seg }} S\right)_{t}=R_{t} \otimes_{K} S_{t}$ for all $t \geq 0$.

One easily sees that the Hilbert function $H_{R \otimes_{\text {seg }} S}(t)=\operatorname{dim}_{K}\left(R \otimes_{\text {seg }} S\right)_{t}$ is given by the product

$$
H_{R \otimes_{s e g} S}(t)=H_{R}(t) \cdot H_{S}(t) .
$$

If $R$ and $S$ are standard graded algebras of dimensions $r$ and $s$, respectively, it follows that the dimension of $R \otimes_{\text {seg }} S$ is $r+s-1$ and that the multiplicity of $R \otimes_{\text {seg }} S$, with respect to the homogeneous maximal ideal, is

$$
e\left(R \otimes_{\text {seg }} S\right)=(r+s-2) ! \cdot \frac{e(R)}{(r-1) !} \cdot \frac{e(S)}{(s-1) !}=\left(\begin{array}{c}
r+s-2 \\
r-1
\end{array}\right) \cdot e(R) \cdot e(S) .
$$

We will prove a general theorem on the existence of linear MCM modules over Segre product rings, showing that such existence is preserved under the operation of Segre product (Theorem 2.4). A central idea of the proof, which will appear again later on, is to use the fact that the Hilbert function of a linear MCM module is equal to the Hilbert function of a free module over a polynomial ring. This often allows one to generalize constructions for polynomial rings to any graded ring which possesses a linear MCM module $M$, by working with the module $M$ instead of the ring.

Theorem 2.4. Let $R$ and $S$ be standard graded algebras over a field $K$ with homogeneous maximal ideals $m$ and $n$, respectively. If $R$ and $S$ each possess a linear MCM module, then the Segre product ring $R \otimes_{\text {seg }} S$ also possesses a linear MCM module. 
Proof. If $R$ and $S$ have linear MCM modules $M$ and $N$, respectively, then the associated graded modules $\operatorname{gr}_{m}(M)$ and $\operatorname{gr}_{n}(N)$ are linear MCMs with all generators in degree zero (Proposition 1.8). From here on we will assume that $M$ and $N$ have these properties.

We may use Noether normalization to choose linear forms $X_{1}, \ldots, X_{r}$ in $R$ and $Y_{1}, \ldots, Y_{s}$ in $S$ such that $R$ and $S$ are graded module-finite extensions of the polynomial rings $A=K\left[X_{1}, \ldots, X_{r}\right]$ and $B=K\left[Y_{1}, \ldots, Y_{s}\right]$, respectively. Since $M$ is a graded linear MCM module over $R$, we know that $M$ is free of rank $c=e_{R}(M)$ as an $A$-module; moreover, $m M=(\underline{X}) M$, which is to say that every linear form of $R$ acts on $M \cong_{A} A^{c}$ by a matrix of linear forms in the $X$ 's. Similarly, $N \cong_{B} B^{d}$, where $d=e_{S}(N)$, and linear forms of $S$ act on $N$ via matrices of linear forms in the $Y$ 's.

For any monomial $\beta=X_{1}^{a_{1}} X_{2}^{a_{2}} \cdots X_{r}^{a_{r}} Y_{1}^{b_{1}} \cdots Y_{s}^{b_{s}}$ in the polynomial ring $K\left[X_{1}, \ldots, X_{r}, Y_{1}, \ldots, Y_{s}\right]$, we denote by

$$
\operatorname{deg}_{X} \beta=a_{1}+a_{2}+\ldots a_{r} \text { and } \operatorname{deg}_{Y} \beta=b_{1}+b_{2}+\ldots b_{s}
$$

the total degrees with respect to the sets of variables $X_{1}, \ldots, X_{r}$ and $Y_{1}, \ldots, Y_{s}$, respectively.

Note that we have a module-finite extension

$$
A \otimes_{\text {seg }} B \subseteq R \otimes_{\text {seg }} S,
$$

and that $A \otimes_{\text {seg }} B$ is isomorphic to the subring of the polynomial ring $K\left[X_{1}, \ldots, X_{r}, Y_{1}, \ldots, Y_{s}\right]$ generated over $K$ by all monomials $\mu$ satisfying $\operatorname{deg}_{X} \mu=$ $\operatorname{deg}_{Y} \mu$. The polynomial ring $K[\underline{X}, \underline{Y}]$ then splits over $A \otimes_{\text {seg }} B$ into a direct sum of finitely generated submodules $U_{\alpha}$, where $U_{\alpha}$ is the submodule of $K[\underline{X}, \underline{Y}]$ generated by all monomials $\mu$ with the property that $\operatorname{deg}_{X} \mu-\operatorname{deg}_{Y} \mu=\alpha$. It is a result of Goto and Watanabe 7] that $U_{\alpha}$ is MCM over $A \otimes_{\text {seg }} B$ for $s>\alpha>-r$.

Although the polynomial ring $K\left[X_{1}, \ldots, X_{r}, Y_{1}, \ldots, Y_{s}\right]$ is not necessarily a module over $R \otimes_{\text {seg }} S$, the free $K\left[X_{1}, \ldots, X_{r}, Y_{1}, \ldots, Y_{s}\right]$-module $M \otimes_{K} N$ of rank $c d$ is an $R \otimes_{\text {seg }} S$-module with module structure induced by those of $R$ on $M$ and of $S$ on $N$. Moreover, since linear forms of $R$ (respectively $S$ ) act on $M \cong A^{c}$ (respectively $N \cong B^{d}$ ) by matrices of linear forms of $K[\underline{X}]$ (resp. $K[\underline{Y}]$ ), we see that $M \otimes_{K} N$ still splits as an $R \otimes_{\text {seg }} S$-module into the direct summands $\left(M \otimes_{K} N\right)_{\alpha}=\left(K[\underline{X}, \underline{Y}]^{(c d)}\right)_{\alpha}=$ the submodule of $M \otimes_{K} N$ generated by the vectors

$$
\left\{\left(v_{1}, \ldots, v_{c d}\right): \text { each } v_{i} \in U_{\alpha}\right\} .
$$

Let us denote $\left(M \otimes_{K} N\right)_{0}$ by $M \otimes_{\text {seg }} N$ and $\left(M \otimes_{K} N\right)_{s-1}$ by $W$. It follows from Goto and Watanabe's result that both $M \otimes_{\text {seg }} N$ and $W$ are MCM modules over $A \otimes_{\text {seg }} B$; hence they are also MCM over $R \otimes_{\text {seg }} S$. Moreover, if we denote the homogeneous maximal ideals of $A \otimes_{\text {seg }} B$ and $R \otimes_{\text {seg }} S$ by $I$ and $J$, respectively, then we know that $I\left(M \otimes_{\text {seg }} N\right)=J\left(M \otimes_{\text {seg }} N\right)$ and $I W=J W$.

It follows, in the first place, that

$$
e\left(J ; M \otimes_{\text {seg }} N\right)=e\left(I ; M \otimes_{\text {seg }} N\right)=c d \cdot\left(\begin{array}{c}
r+s-2 \\
s-1
\end{array}\right),
$$

since $M \otimes_{\text {seg }} N$ is free of rank $c d$ over $A \otimes_{\text {seg }} B$, which has multiplicity equal to $\left(\begin{array}{c}r+s-2 \\ s-1\end{array}\right)$, as in (1). Likewise,

$$
\nu_{R \otimes_{s e g} S}(W)=\nu_{A \otimes_{s e g} B}(W)=c d \cdot \nu_{A \otimes_{s e g} B}\left(U_{s-1}\right) .
$$


Since $U_{s-1}$ is minimally generated over $A \otimes_{\text {seg }} B$ by monomials in the variables $X_{1}, \ldots, X_{r}$ of degree $s-1$, we have

$$
\nu_{A \otimes_{s e g} B}\left(U_{s-1}\right)=\left(\begin{array}{c}
r+s-2 \\
s-1
\end{array}\right) .
$$

Thus, we have shown that the minimal number of generators of $W$ over $R \otimes_{\text {seg }} S$ is equal to the multiplicity of the module $M \otimes_{\text {seg }} N$.

By the same argument as above, we have

$$
e(J ; W)=e(I ; W)=e\left(A \otimes_{\text {seg }} B\right) \cdot \operatorname{rank}_{A \otimes_{s e g} B} W .
$$

It will therefore follow that $W$ is a linear MCM module for $R \otimes_{\text {seg }} S$ if we can show that $W$ and $M \otimes_{\text {seg }} N$ have the same rank as $\left(A \otimes_{\text {seg }} B\right)$-modules. Note that there is certainly an inclusion

$$
M \otimes_{\text {seg }} N \hookrightarrow W
$$

given by multiplication by $X_{1}^{s-1}$ (or indeed any other form of degree $s-1$ in the $X$ 's). Moreover, a typical element of $W$ has the form

$$
w=\left(\sum_{i} f_{1, i}(\underline{X}) \beta_{1 i}, \ldots, \sum_{i} f_{c d, i}(\underline{X}) \beta_{c d, i}\right) \in(K[\underline{X}, \underline{Y}])^{(c d)},
$$

with each $\beta_{j i} \in A \otimes_{\text {seg }} B$ and each $f_{j, i}$ is a form of degree $s-1$. Rewriting, we see that

$$
w=\sum_{j, i} f_{j i}(\underline{X}) \mu_{j i}, \text { where } \mu_{j i}=\beta_{j i} \cdot e_{j} \in M \otimes_{\text {seg }} N .
$$

Thus, it suffices to notice that for any form $f$ of degree $s-1$ in $K[\underline{X}]$, and for any $\mu \in M \otimes_{\text {seg }} N$, one has

$$
f(\underline{X}) \cdot \mu=\frac{f(\underline{X}) Y_{1}^{s-1}}{X_{1}^{s-1} Y_{1}^{s-1}} \cdot X_{1}^{s-1} \mu=\alpha \cdot X_{1}^{s-1} \mu,
$$

where $\alpha$ is in the fraction field $L$ of $A \otimes_{\text {seg }} B$. This implies that the injection of $M \otimes_{\text {seg }} N$ into $W$ given by multiplication by $X_{1}^{s-1}$ induces an isomorphism

$$
L \otimes_{A \otimes_{\text {seg }} B}\left(M \otimes_{\text {seg }} N\right) \stackrel{\sim}{\rightarrow} L \otimes_{A \otimes_{\text {seg }} B} W
$$

of $L$-vector spaces; and we have shown that

$$
\operatorname{rank}_{A \otimes_{s e g} B}\left(M \otimes_{\text {seg }} N\right)=\operatorname{rank}_{A \otimes_{s e g} B}(W),
$$

as required.

Remark 2.5. We remark that, if $R$ and $S$ are domains, and if $M$ and $N$ are linear MCM modules over $R$ and $S$, respectively, then the linear MCM module over $R \otimes_{\text {seg }} S$ produced by Theorem 2.4 has torsion-free rank equal to $\operatorname{rank}_{R}(M)$. $\operatorname{rank}_{S}(N)$ over $R \otimes_{\text {seg }} S$. One way to see this is to note that, just as in the discussion following Definition 2.3, we may easily calculate the Hilbert function of $M \otimes_{\text {seg }} N$ to be given by the product $H_{M}(t) \cdot H_{N}(t)$, and it follows that the multiplicity of $M \otimes_{\text {seg }} N$ is given by

$$
e\left(M \otimes_{\text {seg }} N\right)=\left(\begin{array}{c}
r+s-2 \\
r-1
\end{array}\right) \cdot e(M) \cdot e(N)=e\left(R \otimes_{\text {seg }} S\right) \cdot \operatorname{rank}_{R}(M) \cdot \operatorname{rank}_{S}(N) .
$$

Comparing with the multiplicity of $R \otimes_{\text {seg }} S$ shows that the rank of $M \otimes_{\text {seg }} N$ is indeed $\operatorname{rank}_{R}(M) \cdot \operatorname{rank}_{S}(N)$, and when $R \otimes_{\text {seg }} S$ is a domain, it is shown in the proof of Theorem 2.4 that $M \otimes_{\text {seg }} N$ and the linear MCM module $W$ have the same rank over $R \otimes_{\text {seg }} S$. 
It follows immediately that we may iterate the operation of taking a Segre product and still maintain the existence of a linear MCM module. Note that a Segre product of finitely many graded $K$-algebras $R_{1}, \ldots, R_{t}$ may be defined recursively by

$$
R_{1} \otimes_{\text {seg }} R_{2} \otimes_{\text {seg }} \cdots \otimes_{\text {seg }} R_{t}=R_{1} \otimes_{\text {seg }}\left(R_{2} \otimes_{\text {seg }} \cdots \otimes_{\text {seg }} R_{t}\right) .
$$

Corollary 2.6. Let $R_{1}, \ldots, R_{t}$ be standard graded algebras over a fixed field $K$, and suppose that each possesses a linear MCM module. Then the Segre product ring

$$
R_{1} \otimes_{\text {seg }} R_{2} \otimes_{\text {seg }} \cdots \otimes_{\text {seg }} R_{t}
$$

also possesses a linear MCM module.

The next corollary follows immediately from Corollary 2.6 and the results of Brennan, Herzog, and Ulrich [2] and Backelin, Herzog, and Ulrich [1] on the existence of linear MCM modules.

Corollary 2.7. Let $R_{1}, \ldots, R_{t}$ be standard graded algebras over a fixed field $K$, each of which is either regular, one-dimensional, a two-dimensional Cohen-Macaulay domain, or a complete intersection. Then

$$
R_{1} \otimes_{\text {seg }} R_{2} \otimes_{\text {seg }} \cdots \otimes_{\text {seg }} R_{t}
$$

possesses a linear MCM module.

It should be noted that many of the Segre product rings covered by Corollaries 2.6 and 2.7 are not Cohen-Macaulay and have dimension more than two, and that even the existence of a finitely generated MCM module over such rings was previously unknown. In particular, our results generalize those of Ma on the existence of MCM modules over Segre products of one-dimensional rings [14].

We close this section with the promised result on determinantal rings.

Corollary 2.8. For any $m$ and $n$, and any field $K$, the ring of generic $2 \times 2$ minors

$$
R_{2}(X)=\frac{K\left[X_{i j} ; 1 \leq i \leq m, 1 \leq j \leq n\right]}{\left(X_{i j} X_{k l}-X_{i l} X_{k j}, i<k, j<l\right)}
$$

admits a linear MCM module of rank one.

Proof. All we need to note is that $R_{2}(X)$ is isomorphic to the $K$-subalgebra $R$ of the polynomial ring $K\left[X_{1}, \ldots, X_{m}, Y_{1}, \ldots, Y_{n}\right]$ generated by the monomials

$$
X_{1}^{a_{1}} \cdots X_{m}^{a_{m}} Y_{1}^{b_{1}} \cdots Y_{n}^{b_{n}}
$$

with $\sum a_{i}=\sum b_{j}$, which is just the Segre product of the polynomial rings $K\left[X_{1}, \ldots, X_{m}\right]$ and $K\left[Y_{1}, \ldots, Y_{n}\right]$. Indeed, it is clear that we may map $R_{2}(X)$ onto $R$ by sending $x_{i j}$ to $X_{i} Y_{j}$, since the relations on the $x_{i j}$ in $R_{2}(X)$ are generated by those of the form $x_{i j} x_{k l}-x_{i l} x_{k j}$. Since both rings are known to be domains of the same dimension $m+n-1$ (see e.g. 3]), this map must be an isomorphism.

Theorem 2.4 therefore implies that $R_{2}(X)$ possesses a linear MCM module. Moreover, since the polynomial rings are themselves linear MCM modules of rank one, Remark 2.5 shows that the linear MCM $R_{2}(X)$-module produced by Theorem 2.4 may be chosen to have rank one.

The existence of linear MCM modules for generic rings of minors has thus been proved in the cases of $2 \times 2$ minors and maximal minors. So it is reasonable to hope that one can give a positive answer for all of the rings $R_{r}(X)$ of generic $r \times r$ 
minors. The dimensions and multiplicities of such rings are known (see e.g. [12]): for any $m, n, r>0$, if we set $A=K\left[X_{i j}\right]_{1 \leq i \leq m, 1 \leq j \leq n}$, then

$$
\begin{gathered}
\operatorname{dim}\left(A / I_{r+1}(X)\right)=(m+n-r) \cdot r \quad \text { and } \\
e\left(A / I_{r+1}(X)\right)=\prod_{i=0}^{n-r-1} \frac{(m+i) ! i !}{(r+i) !(m-r+i) !}
\end{gathered}
$$

It is also interesting to note that in the existence proofs for both $R_{2}(X)$ and $R_{m}(X)$, the module constructed is of rank one (this is not explicitly stated in the case of maximal minors, but is quite clear from the proof of [2], Proposition (2.8)). It is therefore conceivable that the rings $R_{r}(X)$ all have rank one linear $\mathrm{MCM}$ modules, and this question should be settled first.

\section{LiNeAr MCM MOdULES OVER VERONESE RiNGS}

In this section we will consider the existence of linear MCM modules over Veronese subrings. The existence of such modules is proved for Veronese subrings of polynomial rings of dimension less than or equal to three. Moreover, in the same way as for Segre products, the proofs can be adapted in order to show that if $R$ is any standard graded algebra of dimension less than or equal to three, and if $R$ possesses a linear MCM module, then all of the Veronese subrings of $R$ likewise possess linear MCM modules. Finally, the proofs of existence entail a classification of graded MCM modules over Veronese subrings which may be of interest in its own right.

The methods for constructing linear MCM modules over Veronese rings of dimension three turn out to yield far-reaching generalizations, as we will see in section 4. Results to be presented there should indicate that the class of Veronese subrings of regular rings is more representative of the class of graded $K$-algebras, in this respect, than might at first be apparent.

We begin by introducing some notation. For any $\mathbb{Z}$-graded module $M$, we denote the $t$-th graded piece of $M$ by $M_{t}$. If $M$ is a module over an $\mathbb{N}$-graded ring, then we denote by $M_{\geq t}$ the submodule

$$
M_{\geq t}=\bigoplus_{i \geq t} M_{i} .
$$

If $S$ is any Noetherian $\mathbb{N}$-graded ring with graded pieces $S_{i}$, and if $t$ is any positive integer, then the $t$-th Veronese subring of $S$ is the subring

$$
S^{(t)}=\bigoplus_{i \in \mathbb{Z}} S_{i t}
$$

Note that $S$ is a finitely generated graded $S^{(t)}$-module, and that $S$ splits into a direct sum

$$
S=\bigoplus_{i=0}^{t-1} S_{i, t},
$$

of $S^{(t)}$-modules, where $S_{i, t}=\bigoplus_{a \in \mathbb{Z}} S_{i+a t}$. 
Likewise, if $M$ is a graded $S$-module, then $M$ becomes an $S^{(t)}$-module via restriction of scalars, and one has an $S^{(t)}$-module splitting

$$
M=\bigoplus_{i=0}^{t-1} M_{i, t},
$$

where the modules $M_{i, t}$ are defined in the analogous way.

Note that if $M$ is a finitely generated MCM $S$-module, then the module-finiteness of $S$ over $S^{(t)}$ implies that $M$ is a finitely generated MCM module over $S^{(t)}$. It follows that all of the direct summands $M_{i, t}$ must be MCM, as well. In particular, this shows that all Veronese subrings of a Cohen-Macaulay ring are Cohen-Macaulay. Finally, it is easy to see that if $S$ is a standard graded domain over a field $K$, then the torsion-free rank over $S^{(t)}$ of any of the modules $S_{i, t}$ is one, whence the torsion-free rank of $S$ over $S^{(t)}$ is $t$.

Next, if $S$ is a graded Cohen-Macaulay ring with graded canonical module $\omega$, we note that the graded canonical module of $S^{(t)}$ is $\omega_{0, t}$ [7. In particular, if $S$ is a $d$-dimensional polynomial ring over a field, with the standard grading, then the graded canonical module of $S$ is $S(-d)$. It follows that the graded Cohen-Macaulay module of $S^{(t)}$ is $S_{-d, t}$ [7].

We will also need to know that the operation $\operatorname{Hom}(-, \omega)$ commutes nicely with the operation of taking a Veronese submodule. This is made explicit by the following lemma, which is proved in 7], chapter 3. (Although the lemma does not appear explicitly, all parts of it may be easily deduced from the cited work.)

Lemma 3.1 (Goto and Watanabe). Let $S$ be a Noetherian graded ring with canonical module $\omega_{S}$, let $M$ be a graded module over the Veronese subring $S^{(t)}$, and set $M^{\prime}=S \otimes_{S^{(t)}} M$. Then for any $i \geq 0$,

$$
\left(\operatorname{Ext}_{S}^{i}\left(M^{\prime}, \omega_{S}\right)\right)_{0, t} \cong \operatorname{Ext}_{S^{(t)}}^{i}\left(M, \omega_{S^{(t)}}\right) .
$$

In particular,

$$
\left(\operatorname{Hom}_{S}\left(M^{\prime}, \omega_{S}\right)\right)_{0, t} \cong \operatorname{Hom}_{S^{(t)}}\left(M, \omega_{S^{(t)}}\right) .
$$

For $M$ a graded module over a graded ring $R$ with canonical module $\omega$, we will denote the module $\operatorname{Hom}_{R}(M, \omega)$ by $M^{\vee}$ (or $M_{R}^{\vee}$, if it is necessary to specify what ring the dual is taken over). One conclusion of the preceding lemma is then that, for $M$ a graded module over the Veronese subring $S^{(t)}$,

$$
M^{\vee}=\left((S \otimes M)^{\vee}\right)_{0, t} .
$$

Although the existence of linear MCM modules over Veronese rings of dimension two follows from the results of [2], the method used here gives a very satisfactory answer in this case:

Proposition 3.2. Let $R=S^{(t)}$ be the t-th Veronese subring of the polynomial ring $S=K[X, Y]$ in two variables over the field $K$. Then any MCM module over $R$ is isomorphic to a direct sum of modules of the form $S_{i, t}$.

Proof. If $M$ is a graded MCM module over $R$, let $Q=\left(S \otimes_{R} M\right)^{\vee \vee}$, which is the reflexivization of the expanded module. Then $Q$ is Cohen-Macaulay, hence free over $S$. But we may also note that, since $M$ is MCM over $R, M=M^{\vee \vee}=Q_{0, t}$ by Lemma 3.1. The conclusion is now apparent. 
Let $S=K[X, Y]$, as above. Since the multiplicity of the Veronese subring $R=S^{(t)}$ is $t$, it follows that the multiplicity of each of the $R$-modules $S_{i, t}$ is also $t$. But for each $0 \leq i<t$ the module $S_{i, t}$ is generated by the $i+1$ monomials of degree $i$ in $X$ and $Y$. We thus obtain:

Corollary 3.3. If $S=K[X, Y]$, then for each $t>0, S_{-1, t}$ is the unique indecomposable linear MCM module over $S^{(t)}$.

The situation becomes more interesting in dimension three, where we actually obtain new results. We first give the classification of MCM modules over the Veronese subrings, then the results on linear MCM modules. As above, $S=K[X, Y, Z]$ will be a polynomial ring in three variables over a field. Note that the graded canonical module of $S$ is $S(-3)$, whence the canonical module of the Veronese subring $S^{(t)}$ is $S_{-3, t}$. In what follows, we denote the $n$-th syzygy module of an $R$-module $M$ by $\operatorname{Syz}^{n}(M)$, or by $\operatorname{Syz}_{R}^{n}(M)$, if the ring needs to be specified.

Proposition 3.4. Let $R=S^{(t)}$ be the $t$-th Veronese subring of $S=K[X, Y, Z]$. Then every finitely generated graded MCM module over $R$ is equal to some $W_{0, t}$, where $W$ is a second syzygy over $S$ of a finitely generated graded module $N$ with the property that $N_{i t}=0$ for any integer $i$ (in particular, $N$ is killed by $(X, Y, Z)^{t-1}$ ).

Proof. As before, let $M$ be a graded MCM module over $R$, and let $Q=$ $\left(S \otimes_{R} M_{R}^{\vee}\right)_{S}^{\vee \vee}$. Since $Q$ has depth at least two over $S, \operatorname{pd}_{S} Q \leq 1$. That is, we have a graded resolution

$$
0 \rightarrow S^{a} \rightarrow S^{b} \rightarrow Q \rightarrow 0
$$

for some nonnegative integers $a$ and $b$. Applying the functor $\operatorname{Hom}(-, S(-3))$ to this sequence, we get a new exact sequence:

$$
0 \rightarrow Q^{\vee} \rightarrow S^{b} \rightarrow S^{a} \rightarrow N \rightarrow 0
$$

where $N=\operatorname{Ext}_{S}^{1}\left(Q, \omega_{S}\right)$ (we have suppressed degree shifts of the free modules throughout).

Since $M$ and $M_{R}^{\vee}$ are MCM $R$-modules, we may apply Lemma 3.1 in order to see that $Q_{0, t}=M^{\vee}$ and $\left(Q^{\vee}\right)_{0, t}=M^{\vee \vee}=M \cdot Q^{\vee}$ is clearly a second syzygy of $N$, so we need only show that $N$ has the required properties. It follows from general results on the Hom and Ext functors that $N$ is graded. Moreover, since $N_{0, t}=\operatorname{Ext}_{R}^{1}\left(M^{\vee}, \omega_{R}\right)$ by Lemma 3.1 , and $M^{\vee}$ is MCM, it follows that $N_{0, t}=0$.

Note that the modules $S_{i}$ are included in the conclusion of the proposition, since free $S$-modules are second syzygies of the zero module.

Remark 3.5. It might seem more natural to say that a MCM module over the Veronese subring is a graded summand of a module of projective dimension at most one over the regular extension ring. This is true, but it is not clear, conversely, that a direct summand of such a module is necessarily MCM. For this reason we have preferred the description in terms of syzygies given above.

For example, the second Veronese subring $R=S^{(2)}$ of $S=K[X, Y, Z]$ has only $R, S_{1,2}$, and $W=\left(\operatorname{Syz}^{2}(K(-1))\right)_{0,2}$ as indecomposable MCM modules. The first two are easily seen not to be linear; but it turns out that the last is. In a similar fashion, one may show the existence of linear MCM modules over all Veronese subrings in dimension three: 
Proposition 3.6. Let $R=S^{(t)}$ be the t-th Veronese subring of the polynomial ring $S=K[X, Y, Z]$ of dimension three. Then $R$ has a linear MCM module of rank two.

Proof. Let $N$ be the kernel of the free $S$-module map

$$
S(-1)^{t+1} \stackrel{A}{\longrightarrow} S^{t-1}
$$

defined by the matrix

$$
A=\left[\begin{array}{cccccccc}
X & Y & Z & 0 & 0 & \cdots & 0 & 0 \\
0 & X & Y & Z & 0 & \cdots & 0 & 0 \\
& & & \cdots & & & & \\
& & & \cdots & & & & \\
0 & 0 & \cdots & 0 & X & Y & Z & 0 \\
0 & 0 & \cdots & 0 & 0 & X & Y & Z
\end{array}\right] .
$$

Now set $M=N_{-1, t}$. We will show that $M$ is a linear MCM module over $R$.

We first note that $(X, Y, Z)^{t-1} S^{t-1}$ is contained in the image of $A$. It is easy to see that $I_{t-1}(A)$, the ideal of $S$ generated by the $(t-1)$-sized minors of $A$, is equal to $(X, Y, Z)^{t-1}$, and that this ideal is contained in the image follows from quite general results on matrices.

It follows that

$$
0 \rightarrow M \rightarrow\left(S_{-2, t}\right)^{t+1} \stackrel{A}{\longrightarrow}\left(S_{-1, t}\right)^{t-1} \rightarrow 0
$$

is a short exact sequence of $R$-modules, and this implies that $M$ is MCM. Moreover, since $\operatorname{rank}_{R} S_{i, t}=1$ for any $i$, we see that $\operatorname{rank}_{R} M=2$, and hence the multiplicity of $M$ is $2 e(R)=2 t^{2}$.

Finally, we know that, for any $l \geq 0$,

$$
\operatorname{dim}_{K} S_{l}=\left(\begin{array}{c}
l+2 \\
l
\end{array}\right)=\frac{(l+1)(l+2)}{2} .
$$

Thus, we have that

$$
\operatorname{dim}_{K}\left(M_{0}\right)=\operatorname{dim}_{K}\left(N_{t-1}\right)=(t+1)\left(\begin{array}{l}
t \\
2
\end{array}\right)-(t-1)\left(\begin{array}{c}
t+1 \\
2
\end{array}\right)=0 .
$$

There are now two ways of seeing that $M$ is linear. In the first place, we may note that, by the preceding remark, $M$ is generated by forms of degree at least $2 t-2$ in $S$. So $m_{R} M=(X, Y, Z)^{t} M$ is generated by forms of degree at least $3 t-2$, all of which must be contained in $\left(X^{t}, Y^{t}, Z^{t}\right)\left(S_{-2, t}\right)^{t+1}$ (by a simple application of the pigeon-hole principle). Then, since (3) is a short exact sequence of MCM modules, and $X^{t}, Y^{t}, Z^{t}$ is a system of parameters of $R$, it follows that $m_{R} M=$ $\left(X^{t}, Y^{t}, Z^{t}\right) M . \quad M$ is thus seen to be linear, as $X^{t}, Y^{t}, Z^{t}$ represents a minimal reduction of $m_{R}=(X, Y, Z)^{t}$.

Alternatively, one may simply calculate that

$$
\operatorname{dim}_{K} M_{1}=\operatorname{dim}_{K}\left(N_{2 t-1}\right)=(t+1) \cdot\left(\begin{array}{c}
2 t \\
2
\end{array}\right)-(t-1) \cdot\left(\begin{array}{c}
2 t+1 \\
2
\end{array}\right)=2 t^{2},
$$

whence $\nu(M) \geq \operatorname{dim}_{K}\left(M_{1}\right)=e(M)$, and $M$ is linear.

As in the case of Segre products, the above proof can be adapted to rings which are not themselves regular, but do possess linear MCM modules, which have Hilbert functions equal to those of free modules over a regular ring. Since the proof is essentially the same, we omit some details. 
Theorem 3.7. Let $S$ be a three-dimensional standard graded algebra over a field $K$ with homogeneous maximal ideal $n$. If $S$ possesses a linear maximal CohenMacaulay module $M$, then any Veronese subring of $S$ also possesses a linear MCM module.

Proof. First, we may replace $M$ by $\operatorname{gr}_{n} M$, which is a graded linear MCM module over $S$, with all generators in degree zero. Now proceed as before, replacing the ring by $M$ : if $R=S^{(t)}$, we define $N$ by the short exact sequence

$$
0 \rightarrow N \rightarrow\left(M_{-2, t}\right)^{t+1} \stackrel{A}{\longrightarrow}\left(M_{-1, t}\right)^{t-1} \rightarrow 0,
$$

where $A$ is the matrix defined in Proposition 3.6, except that here $X, Y, Z$ represent a system of parameters of linear forms in $S$. Note that, since $M$ is linear with all generators in degree zero, $M_{\geq t-1}=(X, Y, Z)^{t-1} M$. Thus the map defined by $A$ surjects onto $\left(M_{-1, t}\right)^{t-1}$ by the same argument as that given in the proof of Proposition 3.6.

Since $M$ is MCM over $S$, the direct summands $M_{i, t}$ must be MCM over $R$, and it follows that the module $N$ is likewise MCM over $R$. Moreover, since the Hilbert function of the module $M$ is given by

$$
\operatorname{dim}_{K} M_{l}=\nu(M) \cdot\left(\begin{array}{c}
l+2 \\
2
\end{array}\right)
$$

we may calculate that

$$
\begin{gathered}
\operatorname{dim}_{K}\left(N_{0}\right)=\nu_{S}(M)\left[(t+1) \cdot\left(\begin{array}{l}
t \\
2
\end{array}\right)-(t-1)\left(\begin{array}{c}
t+1 \\
2
\end{array}\right)\right]=0 \\
\operatorname{dim}_{K}\left(N_{1}\right)=\nu_{S}(M) \cdot\left[(t+1)\left(\begin{array}{c}
2 t \\
2
\end{array}\right)-(t-1)\left(\begin{array}{c}
2 t+1 \\
2
\end{array}\right)\right]=2 t^{2} \cdot \nu_{S}(M) .
\end{gathered}
$$

Thus $\nu_{R}(N) \geq 2 t^{2} \cdot \nu_{S}(M)$. On the other hand,

$$
e(N)=(t+1) e\left(M_{-2, t}\right)-(t-1) e\left(M_{-1, t}\right)=2 t^{2} e(n ; M)=2 t^{2} \nu_{S}(M) .
$$

This completes the proof that $N$ is linear.

The kind of classification we have been carrying out for graded MCM modules over Veronese rings might theoretically be extended to all dimensions, but it quickly becomes complicated and unrevealing. Thus, we will just make a few observations about the case of Veronese rings of dimension four.

As before, if $R$ is the $t$-th Veronese subring of $S=K[W, X, Y, Z]$, and $M$ is a graded MCM over $R$, we let $Q=\left(S \otimes_{R} M_{R}^{\vee}\right)_{S}^{\vee \vee}$. $Q$ then has projective dimension at most two over $S$, so we get short exact sequences:

$$
\begin{aligned}
& 0 \rightarrow U \rightarrow S^{c} \rightarrow Q \rightarrow 0 \\
& 0 \rightarrow S^{a} \rightarrow S^{b} \rightarrow U \rightarrow 0
\end{aligned}
$$

where $U=\operatorname{Syz}_{S}^{1}(Q)$. Applying the functor $\operatorname{Hom}(-, S(-4))$, this gives new exact sequences:

$$
\begin{aligned}
& 0 \rightarrow Q^{\vee} \rightarrow S^{c} \rightarrow U^{\vee} \rightarrow N_{1} \rightarrow 0 \\
& 0 \rightarrow U^{\vee} \rightarrow S^{b} \rightarrow S^{a} \rightarrow N_{2} \rightarrow 0
\end{aligned}
$$

where $N_{1}=\operatorname{Ext}^{1}\left(Q, \omega_{S}\right)$ and $N_{2}=\operatorname{Ext}^{1}\left(U, \omega_{S}\right) \cong \operatorname{Ext}^{2}\left(Q, \omega_{S}\right)$. Since $Q_{0, t}=M^{\vee}$ is MCM, it follows from Lemma 3.1 that each of the modules $N_{1}$ and $N_{2}$ must 
vanish in degrees divisible by $t$. These facts, along with $M=Q_{0, t}^{\vee}$, characterize the graded MCMs over $R$.

The situation is a little more transparent in the special case that $t=2$. In that case, both $N_{1}$ and $N_{2}$ must be killed by the maximal ideal $(W, X, Y, Z)$. It therefore follows from equation (7) that $U^{\vee} \cong S^{m} \oplus\left(\mathrm{Syz}^{2} K\right)^{n}$ for some $m$ and $n$. Then equation (6) implies that $Q^{\vee}$ is a first module of syzygies of $V$ for some module $(W, X, Y, Z) U^{\vee} \subseteq V \subseteq U^{\vee}$. The following lemma characterizes the submodules $V$ of $U^{\vee}$ with this property.

Lemma 3.8. Let $(R, m, K)$ be either a local ring with residue field $K$, or else a standard graded $K$-algebra with homogeneous maximal ideal $m$, and let $M$ be a finitely generated (graded) $R$-module. Suppose that one has an $R$-module $U$ and $n \geq 0$ such that

$$
(m R)^{n} \oplus m M \subseteq U \subseteq R^{n} \oplus M .
$$

Then $U \cong R^{k} \oplus(m R)^{n-k} \oplus M^{\prime}$ for some $k \leq n$ and some submodule $M^{\prime}$ of $M$ with $m M \subseteq M^{\prime}$.

Proof. Let $M^{\prime}=M \cap U$. Since $m M \subseteq M^{\prime}$, we know that $U /\left(m R^{n}+M^{\prime}\right)$ is a $K$ vector space of dimension $k \leq n$. Moreover, by applying a $K$-linear automorphism of $R^{n}$, we may assume that a basis of $U /\left(m R^{n}+M^{\prime}\right)$ is given by the images of elements $\left\{e_{i}+w_{i}: 1 \leq i \leq k\right\}$ in $U$, where we think of $R^{n}$ as $R e_{1}+\cdots+R e_{n}$, and where $w_{i} \in M$ for each $i$. If we let $V$ be the submodule of $U$ generated by $\left\{e_{i}+w_{i}: 1 \leq i \leq k\right\}$, then it is apparent that $V$ is free of rank $n$. What we wish to show is that

$$
U=V \oplus m\left(R e_{k+1}+\cdots+R e_{n}\right) \oplus M^{\prime} .
$$

First we note that the sum on the right is direct. For a relation it would take the form

$$
\left(v_{1}+u_{1}\right)+v_{2}+u_{2}=0,
$$

where $v_{1} \in R e_{1}+\cdots+R e_{k}, v_{2} \in R e_{k+1}+\cdots+R e_{n}, u_{1}, u_{2} \in M$, and $u_{1} \neq 0$ only if $v_{1} \neq 0$. It is then apparent that the relation must be trivial.

Denote the module on the right-hand side of equation (8) by $U^{\prime}$. Clearly $U^{\prime} \subseteq U$. Note that $m M \subseteq M^{\prime} \subseteq U^{\prime}$; thus for $i \leq k$, we have $m \cdot R e_{i} \subseteq U^{\prime}+m \cdot w_{i}=U^{\prime}$. Hence $m\left(R^{n} \oplus M\right) \subseteq U^{\prime}$. Finally, it follows from the choices of $M^{\prime}$ and $V$ that $U / m\left(R^{n}+M\right)=U^{\prime} / m\left(R^{n}+M\right)$. Thus $U=U^{\prime}$, which proves the lemma.

Combining the lemma with the preceding discussion gives the following classification of MCM modules over the second Veronese subring of a polynomial ring of dimension four.

Proposition 3.9. Let $M$ be a graded $M C M$ over $R=S^{(2)}$, where $S=K[W, X, Y, Z]$. Then $M \cong Q_{0,2}$ for some $S$-module

$$
Q \cong S^{m_{1}} \oplus\left(\operatorname{Syz}_{S}^{2} K\right)^{m_{2}} \oplus \operatorname{Syz}_{S}^{1}\left(U^{\prime}\right)
$$

where $m_{1}, m_{2}$, and $n$ are nonnegative integers, and

$$
(W, X, Y, Z) \cdot\left(\mathrm{Syz}^{2} K\right)^{n} \subseteq U^{\prime} \subseteq\left(\mathrm{Syz}^{2} K\right)^{n} .
$$

To a large extent, we already know about the "even" parts of $S$ and $\operatorname{Syz}_{S}^{2} K$, and it is just a matter of computation to show that in dimension four none of these modules are linear. Thus, in looking for a linear MCM module, we might as well restrict our attention to the "new" modules which are the even-degree parts of $\operatorname{Syz}^{1}\left(U^{\prime}\right)$ 
for $(W, X, Y, Z) \cdot\left(\mathrm{Syz}^{2} K\right)^{n} \subseteq U^{\prime} \subset\left(\mathrm{Syz}^{2} K\right)^{n}$. The following proposition shows that it is possible to construct a linear MCM module over $R=(K[X, Y, Z, W])^{(2)}$ in this way. In fact, we will in this case proceed to the more general result, which produces a linear MCM module over the second Veronese subring of any standard graded algebra of dimension four which possesses a linear MCM module.

Proposition 3.10. Let $R$ be a four-dimensional standard graded $K$-algebra with homogeneous maximal ideal $m$, and assume that $R$ possesses a linear MCM module $M$. Then the second Veronese subring of $R$ also has a linear MCM module.

Proof. We may replace $M$ by $\operatorname{gr}_{m} M$, which is still a linear MCM module, and assume that $M$ is graded with all generators in degree zero. Let $W, X, Y, Z$ be a system of parameters of linear forms for $R$. Define the $R$-module $U$ by the short exact sequence

$$
0 \rightarrow U \rightarrow M(-1)^{4} \stackrel{[W, X, Y, Z]}{\longrightarrow} m M \rightarrow 0 .
$$

Since $M$ is linear MCM over $R$, we see that $U_{\text {odd }}$, the $R^{(2)}$-summand of $U$ generated in odd degrees, is MCM over $R^{(2)}$. Moreover, since $M$ is free over $A=$ $K[W, X, Y, Z]$, and since $m M=(W, X, Y, Z) M, U$ is none other than the $A$-module Syz $_{A}^{2} K \otimes_{A} M$, where $A=K[W, X, Y, Z]$.

We know that $N=\mathrm{Syz}_{A}^{2} K$ is generated as an $A$-module by the six vectors

$$
\left[\begin{array}{c}
Y \\
-X \\
0 \\
0
\end{array}\right], \quad\left[\begin{array}{c}
Z \\
0 \\
-X \\
0
\end{array}\right],\left[\begin{array}{c}
W \\
0 \\
0 \\
-X
\end{array}\right],\left[\begin{array}{c}
0 \\
Z \\
-Y \\
0
\end{array}\right],\left[\begin{array}{c}
0 \\
W \\
0 \\
-Y
\end{array}\right],\left[\begin{array}{c}
0 \\
0 \\
W \\
-Z
\end{array}\right]
$$

in $S^{(4)}$ (which we will denote by $e_{1}, e_{2}, \ldots, e_{6}$, ordered as above). Let $V$ be the submodule generated by $e_{1}, e_{2}, e_{3}+e_{4}, e_{5}, e_{6}$. Since

$$
\begin{gathered}
X e_{4}=-Z e_{1}+Y e_{2}, \quad W e_{4}=Z e_{5}-Y e_{6}, \\
Y e_{3}=W e_{1}+X e_{5}, \text { and } Z e_{3}=W e_{2}+X e_{6},
\end{gathered}
$$

we see that $(W, X, Y, Z) \cdot N \subseteq V$.

Now we let $U^{\prime}=V \otimes_{A} M \subseteq U$. Since $m M=(W, X, Y, Z) M$, we also have $m U \subseteq U^{\prime}$, whence $U_{\text {odd }}^{\prime}=U_{\text {odd }}$ is MCM over $R^{(2)}$. We may then define an $R$ module $Q$ by the exact sequence

$$
0 \rightarrow Q \rightarrow M^{5} \rightarrow U^{\prime} \rightarrow 0
$$

where the surjection of $M^{5}$ onto $U^{\prime}$ is the one given by the generators $e_{1}, e_{2}, e_{3}+$ $e_{4}, e_{5}$, and $e_{6}$. Since $U_{o d d}^{\prime}$ and $M$ are MCM over $R^{(2)}$, we see that $Q_{o d d}$ is also MCM over $R^{(2)}$.

By employing a suitable degree shift, we may assume that the generators of $U^{\prime}$ are in degree zero. The Hilbert function of $N=\mathrm{Syz}_{A}^{2} K$ can be easily calculated, and we see that

$$
\operatorname{dim}_{K}\left(U_{1}^{\prime}\right)=\nu(M) \cdot \operatorname{dim}_{K}\left(N_{1}\right)=20 \nu(M) .
$$

Since $M$ is linear, we also have that

$$
\operatorname{dim}_{K}\left(M_{1}^{(5)}\right)=4 \cdot \nu\left(M^{5}\right)=20 \nu(M),
$$

and this shows that $Q_{1}=0$. 
Thus $Q_{\text {odd }}$ is a submodule of $M_{\text {odd }}^{5}$ generated by forms in $M^{5}$ of degree at least three. Since $M$ is linear, we know that

$$
M_{\geq 5}=(W, X, Y, Z)^{5} M \subseteq\left(W^{2}, X^{2}, Y^{2}, Z^{2}\right) M,
$$

and thus

$$
m^{4} M_{o d d}=(W, X, Y, Z)^{4} M_{o d d} \subseteq\left(W^{2}, X^{2}, Y^{2}, Z^{2}\right) M_{o d d} .
$$

Since

$$
0 \rightarrow Q_{\text {odd }} \rightarrow M_{\text {odd }}^{5} \rightarrow U_{\text {odd }}^{\prime} \rightarrow 0
$$

is a short exact sequence of MCM modules, it follows that

$$
m^{2} Q_{o d d} \subseteq\left(W^{2}, X^{2}, Y^{2}, Z^{2}\right) Q_{o d d},
$$

and we see that $Q_{\text {odd }}$ is a linear MCM module over $R^{(2)}$ (note that $W^{2}, X^{2}, Y^{2}, Z^{2}$ is a system of parameters for $\left.R^{(2)}\right)$.

Corollary 3.11. Let $S=K[W, X, Y, Z]$, the polynomial ring in four variables over a field. Then $S^{\left(2^{n}\right)}$ has a linear MCM module for any $n \geq 0$.

The final result gives some indication that linear MCM modules ought to exist in general over Veronese rings of dimension four. However, even the case of $K[W, X, Y, Z]^{(3)}$ remains open.

\section{Approximation Results for General Graded Rings}

So far we have been unable to resolve the question of existence of linear maximal Cohen-Macaulay modules over Veronese rings in any further cases. But the methods used to prove the existence of linear MCM modules over Veronese rings of dimension three can be adapted in order to produce vastly more general results, the strongest of which is to be given in Theorem 4.3. Although the proof is quite technical, the main ideas may be briefly summarized.

First, by employing a matrix similar to that used in the proof of Proposition 3.6, one may show that any Veronese subring of a polynomial ring of dimension $d$ possesses a graded MCM of reduction degree $d-2$. Second, by using a system of parameters of linear forms, one may produce similar modules over the Veronese subrings of any graded $K$-algebra $R$. Little can be said about the actual reduction degrees of these modules, but their behavior will in a certain sense approach that of the modules constructed when $R$ is regular. Finally, if $R$ is a ring of prime characteristic, then by employing the Frobenius endomorphism, one may view modules over certain Veronese subrings of $R$ as $R$-modules via restriction of scalars. It turns out that, in this way, one can produce $R$-modules that are arbitrarily "close" to having reduction degree $d-2$ (Theorem 4.3).

This theorem has produced applications to the Conjecture 1.3 of Lech on the multiplicities of a flat couple of local rings [8]. The argument is also quite interesting in itself, in that it provides a compelling example of how a property of Veronese subrings of regular rings may be generalized in order to prove the existence of an "approximate" property over a much broader class of positively graded rings.

We begin with a result for Veronese subrings of polynomial rings of dimension at least three. The proof is a direct generalization of the argument for the existence of linear MCM modules over Veronese rings of dimension three. 
Proposition 4.1. If $R=S^{(t)}$ is any Veronese subring of a polynomial ring $S=$ $k\left[X_{1}, \ldots, X_{d}\right]$ of dimension $d \geq 3$, then $R$ possesses a rank $d-1$ MCM module with reduction degree at most $d-2$.

Proof. We denote the homogeneous maximal ideal $\left(X_{1}, \ldots, X_{d}\right)$ of $S$ by $m_{S}$ and the homogeneous maximal ideal $\left(X_{1}, \ldots, X_{d}\right)^{t}$ of $R$ by $m_{R}$.

Let $N$ be the kernel of the free $S$-module map

$$
S(-1)^{t+d-2} \stackrel{A}{\longrightarrow} S^{t-1}
$$

defined by the $(t-1) \times(t+d-2)$ matrix

$$
A=\left[\begin{array}{ccccccccc}
X_{1} & X_{2} & \cdots & X_{d} & 0 & 0 & \cdots & 0 & 0 \\
0 & X_{1} & \cdots & X_{d-1} & X_{d} & 0 & \cdots & 0 & 0 \\
& & & & \cdots & & & & \\
& & & & \cdots & & & & \\
0 & 0 & \cdots & 0 & X_{1} & X_{2} & \cdots & X_{d} & 0 \\
0 & 0 & \cdots & 0 & 0 & X_{1} & \cdots & X_{d-1} & X_{d}
\end{array}\right] .
$$

Now set $M=N_{-1, t}$.

As in the dimension three case, $\left(X_{1}, \ldots, X_{d}\right)^{t-1} S^{t-1}$ is contained in the image of $A$, which implies that

$$
0 \rightarrow M \rightarrow\left(S_{-2, t}\right)^{t+d-2} \stackrel{A}{\longrightarrow}\left(S_{-1, t}\right)^{t-1} \rightarrow 0
$$

is a short exact sequence of $R$-modules, and this implies that $M$ is MCM. Moreover, since $\operatorname{rank}_{R} S_{i, t}=1$ for any $i$, we know that $\operatorname{rank}_{R} M=d-1$.

Finally, we know that, for any $l$,

$$
\operatorname{dim}_{K} S_{l}=\left(\begin{array}{c}
l+d-1 \\
l
\end{array}\right)
$$

Therefore,

$$
\operatorname{dim}_{K}\left(M_{0}\right)=\operatorname{dim}_{K}\left(N_{t-1}\right)=(t+d-2)\left(\begin{array}{c}
t+d-3 \\
d-1
\end{array}\right)-(t-1)\left(\begin{array}{c}
t+d-2 \\
d-1
\end{array}\right)=0 .
$$

Thus $M$ is generated by forms of degree at least $2 t-2$ in $S$, so that $m_{R}^{d-2} M=$ $m_{S}^{t(d-2)} M$ is generated by forms of degree at least $d t-2$, all of which must be contained in $\left(X_{1}^{t}, X_{2}^{t}, \ldots, X_{d}^{t}\right)\left(S_{-2, t}\right)^{t+d-2}$. Since (10) is a short exact sequence of MCM modules, and $X_{1}^{t}, \ldots, X_{d}^{t}$ is a system of parameters of $R$, it follows that

$$
m_{R}^{d-2} M \subseteq\left(X_{1}^{t}, X_{2}^{t}, \ldots, X_{d}^{t}\right) M,
$$

proving the proposition.

Now we show how to use the above methods in order to produce a sequence of modules with nice properties for any standard graded algebra over a field $K$ of positive prime characteristic $p$. The assumption on the characteristic allows us to view certain Veronese subrings of $R$ as algebras over $R$, so that modules constructed over the Veronese subring become $R$-modules by restriction of scalars. First it is necessary for us to define what we mean by a sequence of MCM modules with reduction degrees approaching a certain number. 
Definition 4.2. Let $(R, m, K)$ be either a local ring with residue field $K$ or a standard graded $K$-algebra with homogeneous maximal ideal $m$. The asymptotic reduction degree of a sequence $\left\{M_{i}\right\}_{i \in \mathbb{N}}$ of finitely generated MCM $R$-modules is defined to be the least integer $t$ such that, for some ideal $I$ generated by a system of parameters,

$$
\lim _{i \rightarrow \infty} \frac{\ell\left(\left(m^{t}+I\right) M_{i} / I M_{i}\right)}{\ell\left(M_{i} / I M_{i}\right)}=0 .
$$

This says that, in the limit, one may more or less ignore the contribution to the multiplicity $\ell\left(M_{i} / I M_{i}\right)$ of $\left(\left(m^{t}+I\right) M_{i} / I M_{i}\right)$. Note that we can always choose some $t$ so that $m^{t} \subseteq I$; thus the asymptotic reduction degree exists, and is at most $t$, for any sequence of modules $\left\{M_{i}\right\}$.

Theorem 4.3. Suppose that $R$ is a standard graded algebra of dimension $d \geq 3$ over a field $K$ of characteristic $p>0$, with homogeneous maximal ideal $m$. Suppose, moreover, that $R$ possesses a finitely generated graded MCM module $M$. Then $R$ possesses a sequence $\left\{M_{i}\right\}$ of MCM modules whose asymptotic reduction degree is at most $d-2$.

Proof. We first prove the theorem in the case that the coefficient field $K$ is perfect.

Without loss of generality, we may assume that $M_{t}=0$ for all $t<0$. We begin by fixing some notation. First, let the Hilbert polynomial $P_{M}(t)$ associated to the Hilbert function $H_{M}(t)=\operatorname{dim}_{K}\left(M_{t}\right)$ of $M$ be given by

$$
P_{M}(t)=\frac{e}{(d-1) !} t^{d-1}+k t^{d-2}+O\left(t^{d-3}\right) .
$$

Next, fix a minimal reduction $I$ of $m$ generated by a system of parameters of oneforms, and a positive integer $a$ such that $m^{n+a}=I^{n} m^{a}$ for all $n \geq 0$. We may likewise fix an integer $b$ so that $M_{\geq t}=m^{t-b} M_{\geq b}$ for all $t \geq b$. It follows that if we set $c=a+b$, then

$$
M_{\geq t}=I^{t-c} M_{\geq c}
$$

for all $t \geq c$. Moreover, we may note that since

$$
m^{t} M \subseteq M_{\geq t} \subseteq m^{t-b} M
$$

for all $t \geq b, e=e(m ; M)$ (in other words, the multiplicities given by the grading of $M$ and the grading of $\operatorname{gr}_{m}(M)$ are the same).

For each $q=p^{i}$ great enough so that $q>c$ and $H_{M}(t)=P_{M}(t)$ for $t \geq q-2$, we define an $R^{(q)}$-module $W_{i}$ as follows: let

$$
N_{i}=\operatorname{Ker}\left[M(-1)^{q-c+d-2} \stackrel{A}{\longrightarrow} M^{q-c-1}\right],
$$

where $I=\left(X_{1}, \ldots, X_{d}\right)$ and $A$ is the $(q-c-1) \times(q-c+d-2)$ sized matrix:

$$
\left[\begin{array}{ccccccccc}
X_{1} & X_{2} & \cdots & X_{d} & 0 & \cdots & 0 & 0 & 0 \\
0 & X_{1} & \cdots & X_{d-1} & X_{d} & \cdots & 0 & 0 & 0 \\
& & & & \cdots & & & & \\
& & & & \cdots & & & & \\
0 & 0 & 0 & \cdots & X_{1} & X_{2} & \cdots & X_{d} & 0 \\
0 & 0 & 0 & \cdots & 0 & X_{1} & \cdots & X_{d-1} & X_{d}
\end{array}\right]
$$

as in the proof of Proposition 4.1. Now set $W_{i}=\left(N_{i}\right)_{-1, q}$.

If $F: R \rightarrow R$ represents the Frobenius endomorphism, then we may note that $F^{i}$ maps $R$ into $R^{(q)}$ for each $i>0$ and $q=p^{i}$. Thus the $R^{(q)}$-module $W_{i}$ becomes an 
$R$-module by restriction of scalars for $F^{i}$. Moreover, since $I^{q-c-1} M^{q-c-1}$ is in the image of $A$, and since $M_{\geq q-1}=I^{q-c-1} M_{\geq c}$, we see that there is an exact sequence

$$
0 \rightarrow W_{i} \rightarrow\left(M_{-2, q}\right)^{q-c+d-2} \rightarrow\left(M_{-1, q}\right)^{q-c-1} \rightarrow 0
$$

induced by $A$ (recall that $q$ must be sufficiently large). In particular, since the latter two modules are MCM over $R$ via $F^{i}$ (and also finitely generated, since $K$ is perfect), we see that the module $W_{i}$ is a finitely generated MCM $R$-module.

It remains to show that the modules $W_{i}$ satisfy the stipulated numerical condition. First, one can easily see from the Hilbert polynomial $P_{M}(t)$, and from the fact that $m^{q}$ is in the integral closure of $m^{[q]}$, that $e\left(m ; M_{f, q}\right)=q^{d-1} e$ for any $q$ and any $0 \leq f<q$, and we may therefore calculate from the short exact sequence (11) that

$$
e\left(m ; W_{i}\right)=e(d-1) q^{d-1} .
$$

Now, if $\left(W_{i}\right)_{0}=\left(N_{i}\right)_{q-1}$ is the first (potentially) nonzero graded piece of $W_{i}$, we may use the short exact sequence (11) to compute the following:

$$
\begin{aligned}
\operatorname{dim}_{K}\left(\left(W_{i}\right)_{0}\right)= & (q-c+d-2) \cdot P_{M}(q-2)-(q-c-1) \cdot P_{M}(q-1) \\
= & (q-c+d-2)\left[(e /(d-1) !)(q-2)^{d-1}+k(q-2)^{d-2}+O\left(q^{d-3}\right)\right] \\
& -(q-c-1)\left[(e /(d-1) !)(q-1)^{d-1}+k(q-1)^{d-2}+O\left(q^{d-3}\right)\right] .
\end{aligned}
$$

One can easily see that this is a polynomial in $q$ of degree at most $d-1$; but, in fact, one may even calculate the coefficient on $q^{d-1}$ to be

$$
\begin{aligned}
& (e /(d-1) !)(-c+d-2)+(d-1)(e /(d-1) !)(-2)+k \\
& \quad-(e /(d-1) !)(-c-1)-(d-1)(e /(d-1) !)(-1)-k=0 .
\end{aligned}
$$

This shows that $\operatorname{dim}_{K}\left(\left(W_{i}\right)_{0}\right)$ is given by a polynomial $P_{0}(q)$ in $q=p^{i}$ of degree no greater than $d-2$.

Also, one should note that, for any $q$ sufficiently large,

$$
\left(m^{d-1}\right)^{q} \cdot m^{c} \cdot M_{\geq q-2}=M_{\geq d q+c-2}=I^{d q-2} \cdot M_{\geq c} \subseteq I^{[q]} M .
$$

Thus, $\left(m^{d-1}\right)^{[q]} \cdot M_{\geq q-2} \subseteq I^{[q]} M:_{M} m^{c}$. Moreover, note that for any $q=p^{i}$,

$$
\ell\left(\frac{I^{[q]} M:_{M} m^{c}}{I^{[q]} M}\right) \leq r(M) \cdot \ell\left(R / m^{c}\right),
$$

where $r(M)$ is the type of the module $M$ (since the dual is an $R$-module with $r(M)$ generators that is killed by $m^{c}$ ).

We may conclude that the length of

$$
m^{d-1} \cdot\left(M_{-2, q}^{q-c+d-2} / I M_{-2, q}^{q-c+d-2}\right)
$$

is bounded by $\beta \cdot(q-c+d-2)$ for all $q$, where $\beta=r(M) \cdot l\left(R / m^{c}\right)$ is constant. Note that a similar, but easier, argument shows that $m^{d} \cdot M_{-2, q} \subseteq I M_{-2, q}$ for $q \geq c$.

Hence, considering the short exact sequence

$$
0 \rightarrow W_{i} / I W_{i} \rightarrow M_{-2, q}^{q-c+d-2} / I M_{-2, q}^{q-c+d-2} \rightarrow M_{-1, q}^{q-c-1} / I M_{-1, q}^{q-c-1} \rightarrow 0
$$

given by tensoring with $R / I$, we see that

$$
\ell\left(\frac{\left(m^{d-2}+I\right) W_{i}}{I W_{i}}\right) \leq \nu\left(m^{d-2}\right) \cdot \operatorname{dim}_{K}\left(\left(W_{i}\right)_{0}\right)+\beta \cdot(q-c+d-2)
$$


a polynomial in $q$ of degree at most $d-2$. Since the multiplicity of $W_{i}$ is given by a polynomial of degree $d-1$ in $q$, this clearly shows that the sequence of MCM modules $\left\{W_{i}\right\}$ has asymptotic reduction degree at most $d-2$.

All that remains is to reduce the statement of the theorem to the case that the coefficient field $K$ is perfect. To do this, we let $K^{\infty}$ represent the perfect closure of $K$. It is easily seen that the ring $R^{\prime}=K^{\infty} \otimes_{K} R$ with homogeneous maximal ideal $m^{\prime}=m R^{\prime}$ still satisfies all hypotheses of the theorem. Since the coefficient field of $R^{\prime}$ is perfect, we may then conclude, as above, that $R^{\prime}$ possesses a sequence $\left\{M_{i}^{\prime}\right\}$ of MCM modules with asymptotic reduction degree at most $d-2$.

Since $R^{\prime}$ is an affine $K^{\infty}$-algebra, there exists, for each $i$, a finite purely inseparable extension $K_{i}$ of $K$ such that the module $M_{i}^{\prime}$ can be presented over $K_{i}$; in other words, there exists a finitely generated module $M_{i}$ over $R_{i}=K_{i} \otimes_{K} R$ such that $M_{i}^{\prime}=K^{\infty} \otimes_{K_{i}} R_{i}$. It follows from the finiteness of $K_{i}$ over $K$ that $M_{i}$ is a finitely generated $R$-module for each $i$. Moreover, the extensions $R \subseteq R_{i}$ and $R_{i} \subseteq R^{\prime}$ are both faithfully flat, and $m_{R_{i}}=m R_{i}, m^{\prime}=m R^{\prime}=m_{R_{i}} R^{\prime}$. From this we may draw several conclusions:

(1) $M_{i}$ is a MCM $R$-module for each $i$.

(2) If $I$ is a minimal reduction of $m$ (and hence of $m_{R_{i}}$ ), then

$$
\ell_{R}\left(M_{i} / I M_{i}\right)=\left[K_{i}: K\right] \cdot \ell_{R_{i}}\left(M_{i} / I M_{i}\right)=\left[K_{i}: K\right] \cdot \ell_{R^{\prime}}\left(M_{i}^{\prime} / I M_{i}^{\prime}\right) .
$$

(3) Likewise,

$$
\begin{aligned}
\ell_{R}\left(\frac{\left(m^{d-2}+I\right) M_{i}}{I M_{i}}\right) & =\left[K_{i}: K\right] \cdot \ell_{R_{i}}\left(\frac{\left(m^{d-2}+I\right) M_{i}}{I M_{i}}\right) \\
& =\left[K_{i}: K\right] \cdot \ell_{R^{\prime}}\left(\frac{\left(m^{d-2}+I\right) M_{i}^{\prime}}{I M_{i}^{\prime}}\right) .
\end{aligned}
$$

It easily follows that

$$
\lim _{i \rightarrow \infty} \frac{\ell_{R}\left(\left(m^{d-2}+I\right) M_{i} / I M_{i}\right)}{\ell_{R}\left(M_{i} / I M_{i}\right)}=0,
$$

showing that $\left\{M_{i}\right\}$ is a sequence of finitely generated $R$-modules satisfying the conclusion of the theorem.

Remark 4.4. We remark on the case that $R$ is a standard graded algebra over a perfect field of characteristic $p$, of dimension one or two. In this case it is well known that $R$ does possess a graded MCM module $M$. When $\operatorname{dim}(R)=1$, the same arguments as in the proof of the theorem show that $M_{-1, q}$ is a linear MCM module for all sufficiently large $q=p^{e}$.

When $\operatorname{dim}(R)=2$, the argument shows that the modules $M_{-1, q}$ have asymptotic reduction degree less than or equal to one. Moreover, if none of the modules $M_{-2, q}$ is linear, then the same argument as in the proof of Theorem 4.3 can be used to produce short exact sequences

$$
0 \rightarrow W_{i} \rightarrow\left(M_{-2, q}\right)^{q-c} \rightarrow\left(M_{-1, q}\right)^{q-c-1} \rightarrow 0
$$

and to prove that $W_{i}$ is a linear MCM module for all sufficiently large $q$.

Of course, existence of linear MCM modules has been proved for graded rings of dimension less than or equal to two by other means, without the assumption of prime characteristic. 
We believe it is worth stating the result in the case $\operatorname{dim}(R)=3$ separately, in that reduction degree one is equivalent to linearity, and we began with the search for linear MCM modules.

Corollary 4.5. Suppose that $R$ is a three-dimensional standard graded algebra over a field $K$ of characteristic $p>0$. Then $R$ possesses a sequence of MCM modules $M_{i}$ with the property that

$$
\lim _{i \rightarrow \infty} \frac{e\left(M_{i}\right)}{\nu\left(M_{i}\right)}=1
$$

Proof. If $P$ is a homogeneous minimal prime of $R$ with $\operatorname{dim}(R / P)=3$, then it clearly suffices to produce such a sequence of modules over $R / P$. But it follows from a theorem of Peskine and Szpiro [16] that $R / P$ possesses a graded MCM module of positive rank. We may now simply apply Theorem 4.3 with $d=3$.

\section{REFERENCES}

1. J. Backelin, J. Herzog, and B.Ulrich, Linear maximal Cohen-Macaulay modules over strict complete intersections, J. of Pure and Appl. Algebra 71 (1991), 187-202. MR 92g:13011

2. J. Brennan, J. Herzog, and B. Ulrich, Maximally generated Cohen-Macaulay modules, Math. Scand. 61 (1987), 181-203. MR 89j:13027

3. W. Bruns and J. Herzog, Cohen-Macaulay rings, Cambridge Studies in Advanced Mathematics, 39, Cambridge University Press, Cambridge, 1993. MR 95h:13020

4. J. Eagon and M. Hochster, Cohen-Macaulay rings, invariant theory, and the generic perfection of determinantal loci, Amer. J. Math. 93 (1971), 1020-1058. MR 46:1787

5. J. Eagon and D.G. Northcott, Generically acyclic complexes and generically perfect ideals, Proc. Royal Soc., Series A 299 (1967), 147-172. MR 35:5435

6. D. Eisenbud and F.-O. Schreyer (App. by J. Weyman), Resultants and Chow forms via exterior syzygies, J. Amer. Math. Soc. 16 (2003), 537-579.

7. S. Goto and K. Watanabe, On graded rings, I, J. Math. Soc. Japan 30 (1978), 179-213. MR 81m:13021

8. D. Hanes, Special conditions on maximal Cohen-Macaulay modules, and applications to the theory of multiplicities, Thesis, University of Michigan, 1999.

9. _ Length approximations for independently generated ideals, J. Algebra 237 (2001), 708-718. MR 2001m:13006

10. M. Hochster, Big Cohen-Macaulay modules and algebras and embeddability in rings of Witt vectors, Queen's Papers Pure and Appl. Math. 42 (1975), 106-195. MR 53:407

11. - Topics in the homological theory of modules over commutative rings, CBMS Regional Conference Series in Mathematics, No. 24. American Mathematical Society, Providence, RI, 1975. MR 51:8096

12. C. Huneke and M. Miller, A note on the multiplicity of Cohen-Macaulay algebras with pure resolutions, Canadian J. of Math. 37 (1985), 1149-1162. MR 87d:13024

13. C. Lech, Note on multiplicities of ideals, Arkiv for Math. 4 (1960), 63-86. MR 25:3955

14. F. Ma, Splitting in integral extensions, Cohen-Macaulay modules and algebras, J. Algebra 116 (1988), 176-195. MR 89e:13010

15. D.G. Northcott and D. Rees, Reductions of ideals in local rings, Proc. Camb. Phil. Soc. 50 (1954), 145-158. MR 15:596a

16. C. Peskine and L. Szpiro, Notes sur un air de H. Bass, Unpublished preprint, Brandeis University.

17. B. Ulrich, Gorenstein rings and modules with high numbers of generators, Math. Z. 188 (1984), 23-32. MR 85m:13021

School of Mathematics, University of Minnesota, Minneapolis, Minnesota 55455

E-mail address: hanes@math.umn.edu

Current address: Neuro-otology Research, Legacy Research Center, 1225 NE 2nd Avenue,

Portland, Oregon 97232

E-mail address: douglash@neurotology.org 